ORIGINAL ARTICLE

\title{
EVALUATION OF THE ROLE OF BEDSIDE LUNG ULTRASOUND VERSUS CHEST X-RAY IN CRITICALLY ILL PATIENTS
}

\author{
Nermeen Mohammed Ali ${ }^{1 *}$, Hassan M A Maguid ${ }^{1}$, Neven M Gamil ${ }^{1}$, Mohamed M Tawfeek ${ }^{1}$, \\ Sameh Saber Hegab ${ }^{2}$ \\ ${ }^{1}$ Anesthesia and Surgical intensive care Department, Faculty of Medicine, Zagazig University, \\ Zagazig, Egypt \\ ${ }^{2}$ Radiology Department, Faculty of Medicine, Zagazig University, Zagazig, Egypt
}

\section{*Corresponding author: \\ Nermeen Mohammed Ali \\ Anesthesia and Surgical intensive \\ care Department, Faculty of \\ Medicine, Zagazig University, \\ Zagazig, Egypt. \\ dr_nermo83@hotmail.com}

$\begin{array}{ll}\text { Submit Date } & 2019-03-27 \\ \text { Revise Date } & 2019-05-01 \\ \text { Accept Date } & 2019-05-06\end{array}$

\section{INTRODUCTION}

ritical illness is a life-threatening
condition that can result in significant
morbidity or mortality. All clinical staff has
an important role in implementing an
effective "Chain of Response" that includes
accurate recording of vital signs with
recognition and interpretation of abnormal
values, patient assessment and appropriate
intervention ${ }^{(1)}$. Good outcomes rely on rapid
diagnosis and definitive treatment. All
physicians should possess the skills to

\begin{abstract}
Background: Although chest X-ray is the main imaging approach in many settings, many limitations for it exist. Ultrasound has quite similar performances to CT with many advantages. Methods: From January 2017 till May 2018, a prospective cohort study conducted in emergency ICU at Zagazig university hospitals including 124 critically ill patients older than 18 years with respiratory distress, cough, fever, or hypoxemia. We excluded from the study pregnant females, patients with massive chest wall emphysema or hematoma, morbidly obese and finally patients with risk of transportation. All patients underwent thorough physical examination, history, laboratory investigations \& Chest radiology (X-rays, chest ultrasound \& CT). We measured the sensitivity and specificity of chest ultrasound and chest X-rays in comparison with CT with measurement of the learning curve of chest US. Results: 124 patients were assessed for eligibility. 24 patients were excluded for different causes and 100 patients (69 males \& 31 females) completed the study with mean age of $49.22 \pm 11.52$ years. Regarding all study population, whatever diagnosis, sensitivity and specificity of chest ultrasound and chest X-rays were $91.4 \%, 98.3 \%$ and $61.7 \%, 96.2 \%$ respectively. Concordance of the results of ultrasound with results of X-rays and clinical diagnosis increased sensitivity, specificity and overall accuracy to highly comparable results with chest computed tomography. Sensitivity, specificity and accuracy of chest ultrasound increased with time and with number of patients. Conclusions: Chest ultrasound is reliable, quick, bedside, low-cost, non-invasive, non-ionizing, more accurate, and easily educated for early detection of chest diseases and their follow up.
\end{abstract}

Keywords: Critically ill, lung ultrasound, BLUE protocol recognize the critically ill patient and investigate appropriate initial management ${ }^{(2)}$. Lung imaging in critically ill patients was performed traditionally either by bedside chest radiography (CXR) or thoracic computed tomography (CT). Both techniques have limitations which constrain their usefulness. Critically ultrasound, apparently a recent field, is in fact the outcome of a slow process, initiated since 1946. The lung was traditionally not considered as a part of ultrasound, now it is included as a priority in 
the critical ultrasound ${ }^{(3)}$. The possibility of exploring the lung using bedside ultrasound is gaining popularity among intensivists. The Bedside Lung Ultrasonography in Emergency department (BLUE-protocol) is an exclusive diagnostic ultrasound approach intended to be combined with simple clinical data. It proposes a step-by-step analysis, which can be achieved within three minutes ${ }^{(4)}$. The aim of this study is to compare the lung ultrasound in diagnosis of the majority of pathologic pulmonary problems in ICU with bedside chest radiography.

\section{METHODS}

This is a prospective cohort study conducted in emergency ICU at Zagazig university hospitals including critically ill patients older than 18 years of both sexes presented with or newly developed respiratory distress, cough with fever, purulent expectoration, or hypoxemia from January 2017 till May 2018. Respiratory distress included, but not limited to, patients in whom ventilation was indicated due to respiratory failure, impending failure, hypoxemia despite conventional oxygen therapy, and troubleshooting of the mechanically ventilated patient especially if there is high probability of pneumothorax. We excluded from the study pregnant females, patients with inapplicable lung ultrasound e.g massive chest wall emphysema or hematoma, heavy dressing and morbidly obese and finally patients with risk of transportation for CT. This work has been carried out in accordance with The Code of Ethics of the World Medical Association (Declaration of Helsinki) for studies involving humans. After institutional ethics committee approval and an informed written consent were taken from the patient or from his relatives, if not aware. Four pathologic conditions were evaluated by each radiological imaging technique: pneumothorax, pleural effusion, lung consolidation and pulmonary edema. Each hemi-thorax was evaluated for absence or presence of those conditions. All patients of the study were subjected to thorough medical history taking, clinical examination and laboratory testing. All patients were subjected to lung ultrasound, chest X-ray and chest CT scan within a time limit of four hours for logistic reasons.
Chest ultrasonography: Siemens Acuson x300 ultrasound was used in this study using two probes (phased array low frequency 5 $\mathrm{MHz}$ probe mainly and a linear high frequency $7-10 \mathrm{MHz}$ probe occasionally) using the gray-scale (B-mode) and time motion (M-mode). Patients were examined posteriorly in a semi lateral position and anteriorly in a supine position. Lung ultrasound was done by the same operator and revised by expert radiologist in lung ultrasonography who gave the final diagnosis. Both the operator and the expert radiologist were oriented by the clinical situation of the patient and unaware of the other chest radiography findings. Three standardized points were examined. Determination of these points can be achieved by two hands touching each other with thumbs excluded on the patient chest (the upper hand touching the clavicle). The upper point is at the middle of the upper hand, while the lower point is at the middle of the lower palm. The third point is called PLAPS (postero-lateral alveolar or pleural syndrome) point and is by transection of a horizontal plane at the level of the lower BLUE point and a vertical line at the posterior axillary line.

Three signs with dual answers were assessed in each of the previously mentioned points, as follow: A lines (repetitive horizontal artifact arising from the pleural line generated by subpleural air that blocks ultrasound waves) or B Lines (artifact with seven features: A hydroaeric comet-tail artifact, arising from the pleural line, hyperechoic, well defined, spreading up indefinitely, erasing A lines, and moving with lung sliding when lung sliding is present) and absent or present lung sliding or alveolar consolidation and/or pleural effusion. According to the BLUE protocol, combination of these signs results in eight profiles. These profiles are the A-profile (associates anterior lung-sliding with A-lines), the A'-profile (an A-profile with abolished lung-sliding), the B-profile (associates anterior lung-sliding with lung-rockets), the B'-profile (a B-profile with abolished lungsliding), the C-profile (indicates anterior lung consolidation, regardless of size and number), the A/B profile (a half A-profile at one lung, a half B-profile at another), the PLAPS-profile 
(designates Postero-lateral Alveolar and/or Pleural Syndrome), and the nude profile (is an A-profile with no DVT and no PLAPS).

Chest X-rays: Using Arab precise industries company (APiC) MR300 portable X-ray device, antero-posterior chest radiography was done with the patient in supine position. The evaluation of chest X-rays was performed by a radiologist unaware of the lung ultrasound and CT findings. Different lung pathologies were defined using the nomenclature committee of the Fleischner society terminology ${ }^{(5)}$.

Chest computed tomography (CT): A lowdose CT scan without contrast using Toshiba Prime Aquilion - 64 slice high speed device was ordered for all patients to confirm diagnosis. CT scan was done using $120 \mathrm{kv}$, 20-40 mA, and reconstructed layer thickness of $4 \mathrm{~mm}$ and effective radiation dose of 0.4 $\mathrm{mSv}$. CT scan was done while the patient was in supine position from the apex of the thorax to the lung bases, lung images were displayed in both lung and soft tissue window. Different lung pathologies were defined using the terminology of the nomenclature committee of the Fleischner society ${ }^{(5)}$.

Primary goal of this study is measuring the sensitivity and specificity of chest ultrasound and chest X-rays in comparison with chest $\mathrm{CT}$. While, the goal is measuring of the learning curve of chest ultrasonography.

As the average number of cases with inclusion criteria is about 8 cases per month in a study period of one year, sample size was calculated with using a priori test with an effect size of 0.5 for the Wilcoxon signedrank test with an error protection of 0.05 provided $80 \%$ power for the sample size of about 100 cases according to approval of IRB committee, Zagazig University.

\section{Statistical analysis}

The collected data were organized, tabulated and statistically analyzed, using statistical package for social science (SPSS) version 19 (SPSS Inc, Chicago, USA). Mean, standard deviation, frequency and percentage were used as descriptive. Sensitivity, specificity, positive predictive value, negative predictive value, positive likelihood ratio, negative likelihood ratio, and accuracy were used as measurements of validity for chest X-rays and chest ultrasonography results in comparison to $\mathrm{CT}$ chest. The latter was regarded as the standard reference.

\section{RESULTS}

In this prospective study 124 patients was assessed for eligibility. Twenty four patients were excluded for different causes (14 patients: died before doing CT, 6 patients: can't be transferred to $\mathrm{CT}$, and 4 patients: $\mathrm{CT}$ was done after 4 hours from US)(Figure 1) and 100 patients completed the study. Sixty nine of them were males and 31 were females with mean age of $49.22 \pm 11.52$ years. The rest of patient characteristics were shown in (Table 1).

As regard pleural effusion, it was diagnosed by clinical examination in $30(30 \%)$ patients, while it was diagnosed with chest ultrasound, chest X-rays, and chest CT in 63 (63\%), 49 $(49 \%)$ and $65(65 \%)$ patients respectively (Table 2, 3 \& Figure 1, 2A). In diagnosing pleural effusion, sensitivity and specificity of chest ultrasound and chest X-rays in comparison to chest CT were $95.4 \%, 97.1 \%$ and $70.7 \%, 91.45 \%$ respectively. With combination of the results of chest ultrasound, chest X-rays and clinical examination, sensitivity and specificity were $98.5 \%$ and $100 \%$ respectively (Table 4 ).

Regarding pneumothorax, it was diagnosed by clinical examination in $11(11 \%)$ patients, while it was diagnosed with chest ultrasound, chest X-rays, and chest CT in 29 (29\%), 18 $(18 \%)$, and $32(32 \%)$ patients respectively (Table 2, 3 \& Figure 1, 2B). In diagnosing pneumothorax, sensitivity and specificity of chest ultrasound and chest X-rays in comparison to chest CT were $87.5 \%, 98.5 \%$ and $53.1 \%, 98.5 \%$ respectively. Sensitivity and specificity of combined results of chest ultrasound, chest X-rays and clinical examinations were $93.7 \%$ and $98.5 \%$ respectively (Table 4 ).

As for pneumonia, it was diagnosed by clinical examination in combination with laboratory findings in 29 (29\%) patients, while it was diagnosed with chest ultrasound, chest X-rays, and chest CT in 51 (51\%), 38 $(38 \%)$, and $56(56 \%)$ patients respectively (Table 2, 3 \& Figure 1, 2C). In diagnosing pneumonia, sensitivity and specificity of chest ultrasound and chest X-rays in comparison to 
chest CT were $89.3 \%, 97.7 \%$ and $60.7 \%$, $90.9 \%$ respectively. Sensitivity and specificity of combined results of chest ultrasound, chest $\mathrm{X}$-rays and clinical examinations were $94.6 \%$ and $100 \%$ respectively (Table 4 ).

Regarding pulmonary edema, it was diagnosed by clinical examination in $5(5 \%)$ patients, while it was diagnosed with chest ultrasound, chest X-rays, and chest CT in 9 (9\%), 4 (4\%), and $9(9 \%)$ patients respectively (Table 2, $3 \&$ Figure $1,2 \mathrm{D}$ ). In diagnosing pulmonary edema, sensitivity and specificity of chest ultrasound and chest Xrays in comparison to chest $\mathrm{CT}$ were $88.9 \%$, $98.9 \%$ and $33.3 \%$, $98.9 \%$ respectively. Sensitivity and specificity of combined results of chest ultrasound, chest X-rays and clinical examinations were $100 \%$ and $100 \%$ respectively (Table 4).

Regarding all population of the study, whatever diagnosis, sensitivity and specificity

Table 1. Patient demographics

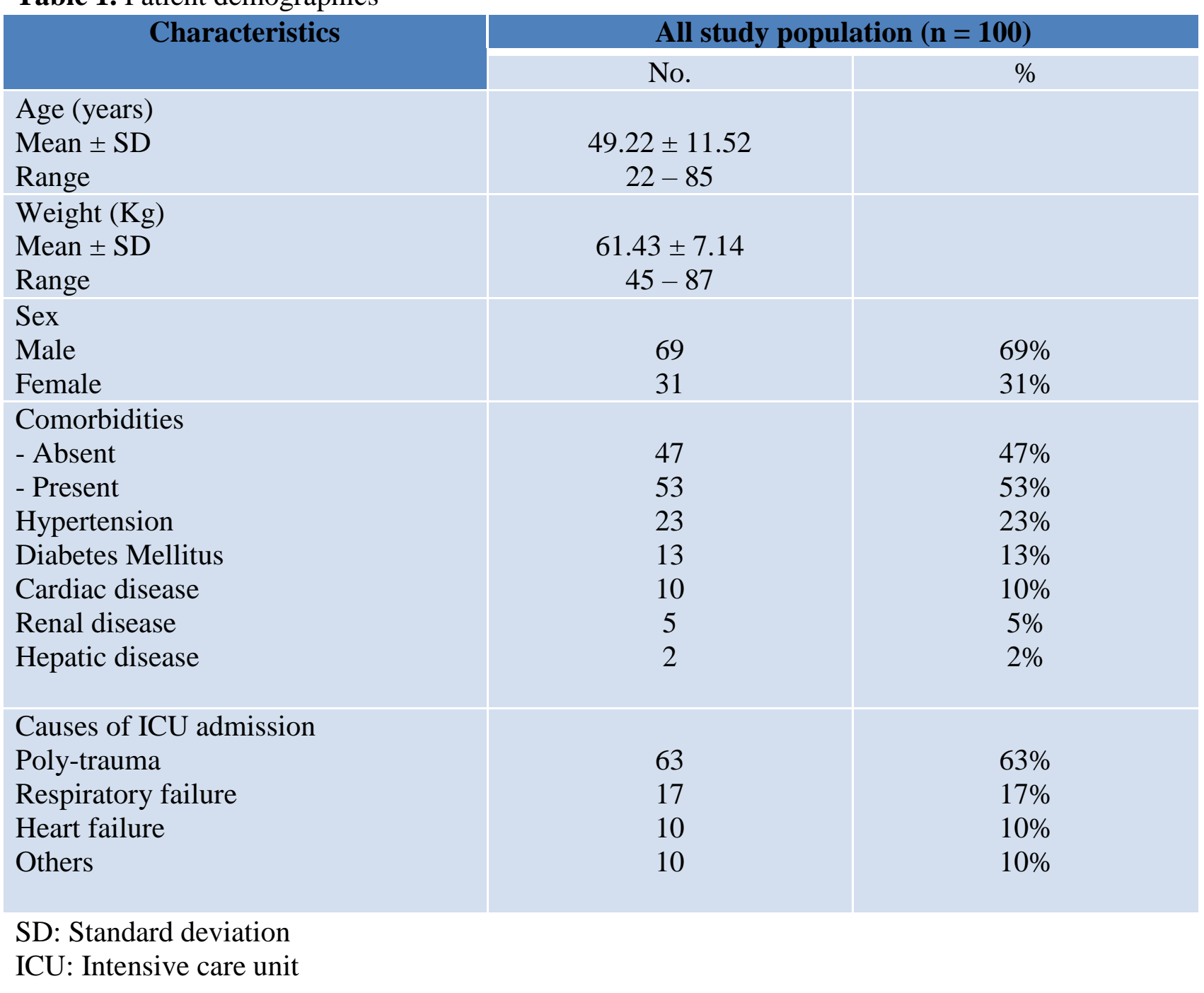

SD: Standard deviation

ICU: Intensive care unit of chest ultrasound and chest X-rays were respectively. Concordance of the results of chest ultrasound with results of chest X-rays and clinical diagnosis increased sensitivity, specificity and overall accuracy to highly comparable results with chest computed tomography (Table 4).

Sensitivity, specificity and accuracy of chest ultrasound, in our study, increased with time and with number of patients. In the first 30 cases, the overall accuracy of chest ultrasound increased rapidly to reach $80.3 \%$. In the next 30 cases, slow increase in the overall accuracy occurred to reach $97.2 \%$. A plateau occurred after that in the next 20 cases. At the end of the study, the overall accuracy of chest ultrasound reached $95.5 \%$. We can use the change in accuracy of chest ultrasound by time as an indicator for the learning curve of chest ultrasound (Figure 3). $91.4 \%, \quad 98.3 \%$ and $61.7 \%, \quad 96.2 \%$

\section{All study population $(n=100)$}


Table 2:Results lung ultrasound and chest X-rays compared to chest CT

\begin{tabular}{|c|c|c|c|}
\hline Pathology & US/CXR & $\mathrm{CT}+$ & CT- \\
\hline Pleural effusion & $\begin{array}{l}\mathrm{US}+ \\
\mathrm{US}- \\
\mathrm{CXR}+ \\
\mathrm{CXR}-\end{array}$ & $\begin{array}{c}62 \\
3 \\
46 \\
19\end{array}$ & $\begin{array}{c}1 \\
34 \\
3 \\
32\end{array}$ \\
\hline Pneumothorax & $\begin{array}{l}\mathrm{US}+ \\
\mathrm{US}- \\
\mathrm{CXR}+ \\
\mathrm{CXR}-\end{array}$ & $\begin{array}{c}28 \\
4 \\
17 \\
15\end{array}$ & $\begin{array}{c}1 \\
67 \\
1 \\
67\end{array}$ \\
\hline Pneumonia & $\begin{array}{l}\mathrm{US}+ \\
\mathrm{US}- \\
\mathrm{CXR}+ \\
\mathrm{CXR}-\end{array}$ & $\begin{array}{c}50 \\
6 \\
34 \\
22\end{array}$ & $\begin{array}{c}1 \\
43 \\
4 \\
40\end{array}$ \\
\hline Pulmonary edema & $\begin{array}{l}\mathrm{US}+ \\
\mathrm{US}- \\
\mathrm{CXR}+ \\
\mathrm{CXR}-\end{array}$ & $\begin{array}{l}8 \\
1 \\
3 \\
6\end{array}$ & $\begin{array}{c}1 \\
90 \\
1 \\
90\end{array}$ \\
\hline
\end{tabular}

US: Ultrasound

CXR: Chest X-rays

CT: Computed tomography

Table 3: Ultrasound lung profiles in different diseases

\begin{tabular}{|l|l|c|}
\hline Diagnosis & Profile & No. $(\%)$ \\
\hline Pleural effusion & A profile + PLAPS & $63(100 \%)$ \\
\hline Pneumothorax & A' profile + Lung point & $29(100 \%)$ \\
\hline Pneumonia & A/B profile & $3(6 \%)$ \\
& C profile & $5(10 \%)$ \\
& A profile + PLAPS & $31(60 \%)$ \\
\hline Pulmonary edema & B' profile & $12(24 \%)$ \\
\hline
\end{tabular}

PLAPS: Postero-lateral alveolar or pleural syndrome

Table 4: Sensitivity, Specificity \& Accuracy in diagnosing chest diseases

\begin{tabular}{|c|c|c|c|c|c|c|c|c|}
\hline Disease & Radiology & $\begin{array}{c}\text { Sensitivity } \\
\%\end{array}$ & $\begin{array}{c}\text { Specificity } \\
\%\end{array}$ & $\begin{array}{l}\text { PPV } \\
\%\end{array}$ & $\begin{array}{c}\text { NPV } \\
\%\end{array}$ & LR+ & LR- & $\begin{array}{c}\text { Accuracy } \\
\%\end{array}$ \\
\hline \multirow{3}{*}{$\begin{array}{l}\text { Pleural } \\
\text { Effusion }\end{array}$} & Ultrasound & $95.4 \%$ & $97.1 \%$ & $98.4 \%$ & $91.8 \%$ & 32.9 & 0.047 & $96 \%$ \\
\hline & X-rays & $70.7 \%$ & $91.45 \%$ & $93.8 \%$ & $62.7 \%$ & 8.27 & 0.32 & $78 \%$ \\
\hline & Combination & $98.5 \%$ & $100 \%$ & $100 \%$ & $97.2 \%$ & & 0.015 & $99 \%$ \\
\hline \multirow[t]{3}{*}{ Pneumothorax } & Ultrasound & $87.5 \%$ & $98.5 \%$ & $96.5 \%$ & $94.3 \%$ & 58.3 & 0.063 & $95 \%$ \\
\hline & X-rays & $53.1 \%$ & $98.5 \%$ & $94.4 \%$ & $81.7 \%$ & 35.4 & 0.476 & $84 \%$ \\
\hline & Combination & $93.7 \%$ & $98.5 \%$ & $96.8 \%$ & $97.1 \%$ & 62.4 & 0.027 & $97 \%$ \\
\hline \multirow[t]{3}{*}{ Pneumonia } & ultrasound & $89.3 \%$ & $97.7 \%$ & $98 \%$ & $87.8 \%$ & 38.8 & 0.11 & $93 \%$ \\
\hline & $\mathrm{X}$-rays & $60.7 \%$ & $90.9 \%$ & $89.4 \%$ & $64.5 \%$ & 6.67 & 0.43 & $74 \%$ \\
\hline & Combination & $94.6 \%$ & $100 \%$ & $100 \%$ & $93.6 \%$ & & 0.054 & $97 \%$ \\
\hline \multirow{3}{*}{$\begin{array}{l}\text { Pulmonary } \\
\text { Edema }\end{array}$} & ultrasound & $88.9 \%$ & $98.9 \%$ & $88.9 \%$ & $98.9 \%$ & 80.81 & 0.112 & $98 \%$ \\
\hline & X-rays & $33.3 \%$ & $98.9 \%$ & $75 \%$ & $93.7 \%$ & 30.27 & 0.674 & $93 \%$ \\
\hline & Combination & $100 \%$ & $100 \%$ & $100 \%$ & $100 \%$ & & 0 & $100 \%$ \\
\hline \multirow{3}{*}{$\begin{array}{l}\text { All study } \\
\text { population }\end{array}$} & ultrasound & $91.4 \%$ & $98.3 \%$ & $97.3 \%$ & $94.4 \%$ & 53.76 & 0.087 & $95.5 \%$ \\
\hline & X-rays & $61.7 \%$ & $96.2 \%$ & $91.7 \%$ & $78.7 \%$ & 16.23 & 0.398 & $82.2 \%$ \\
\hline & Combination & $96.3 \%$ & $99.1 \%$ & $98.7 \%$ & $97.5 \%$ & 107 & 0.037 & $98.2 \%$ \\
\hline
\end{tabular}

PPV: Positive predictive value

NPV: Negative predictive value

$\mathrm{LR}+$ : likelihood ratio of a positive test

LR-: likelihood ratio of a negative test 


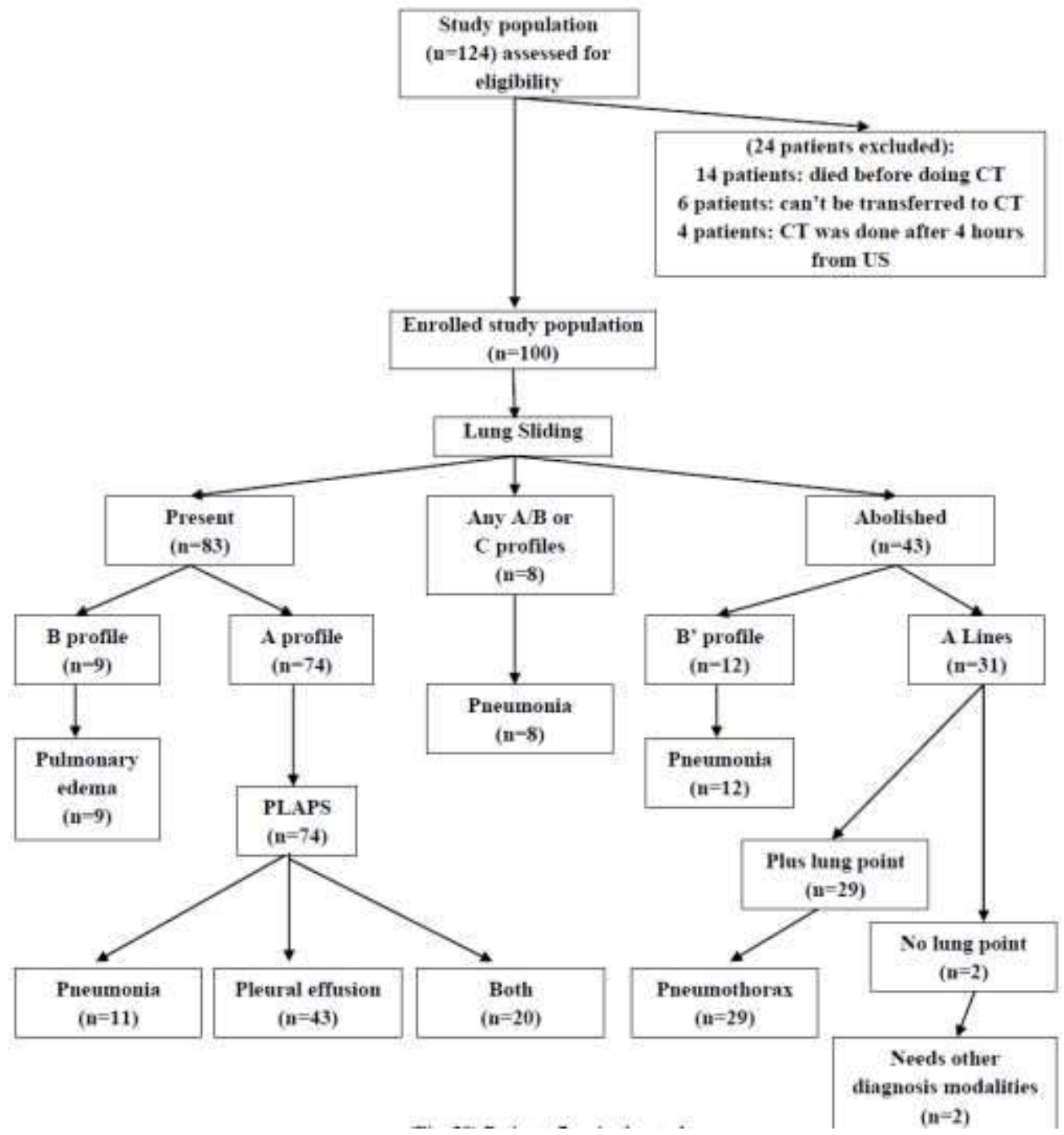

The A-profile: associates anterior lung-sliding with A-lines.

The $A$ '-profile: is an A-profile with abolished lung-sliding.

The B-profile: associates anterior lung-sliding with lung-rockets.

The B'-profile: is a B-profile with abolished lung-sliding.

The $C$-profile: indicates anterior lung consolidation.

The A/B profile: is a half A-profile at one lung, a half B-profile at another.

The PLAPS-profile: designates Postero-lateral Alveolar and/or Pleural Syndrome.

Fig. 1. Patient flow in the study 


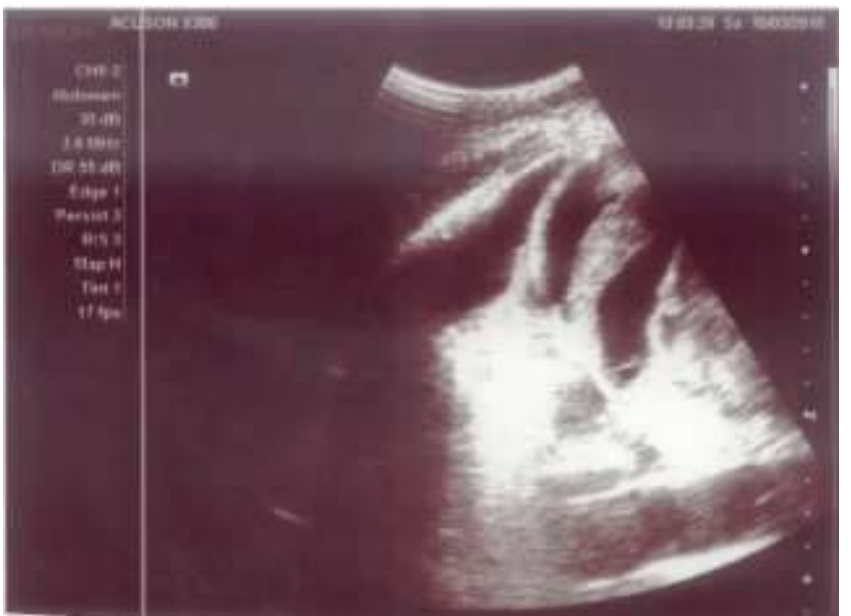

A

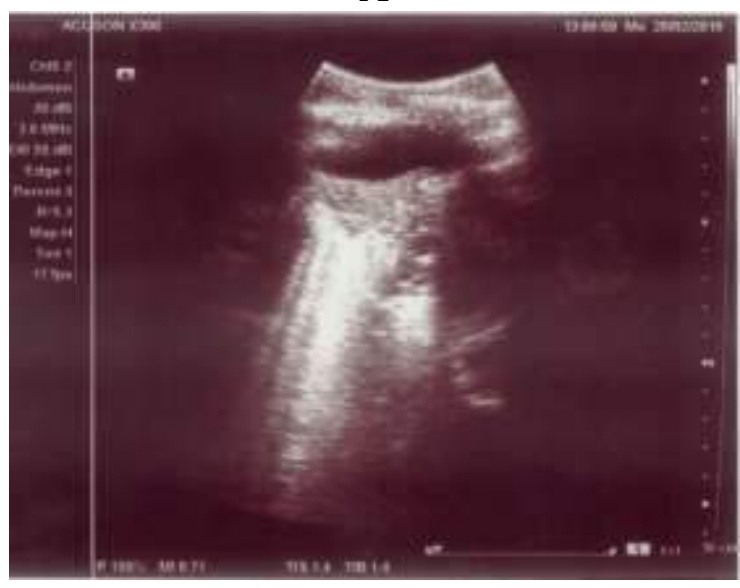

C

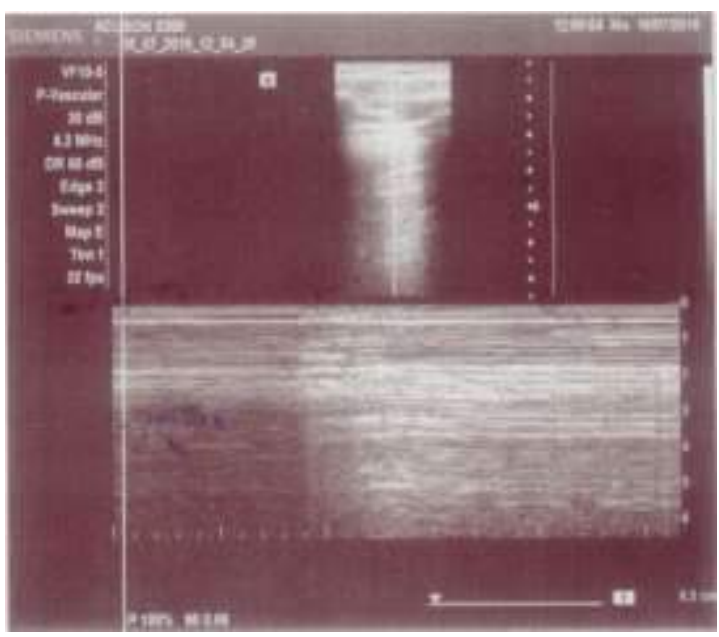

B

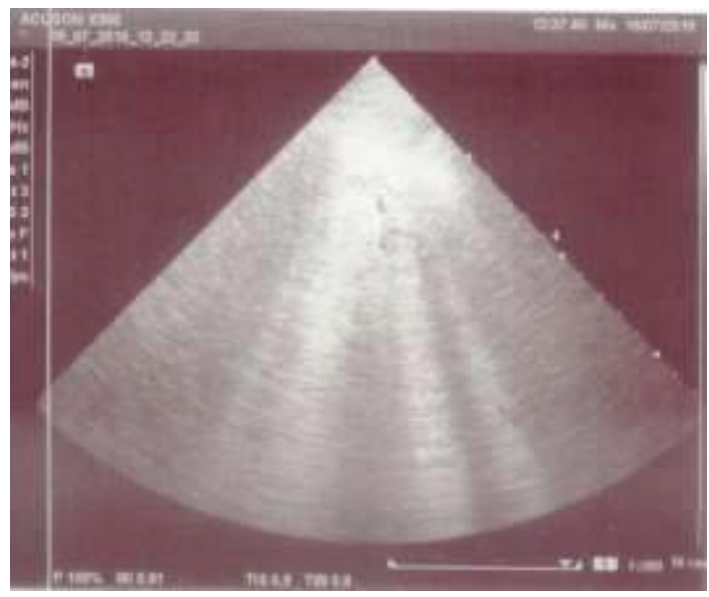

D

Fig. 2.Lung ultrasound pictures from our patients: A: Septated pleural effusion, B: Lung point of Pneumothorax, C: Shred sign of pneumonia, D: B-profile of Pulmonary edema.

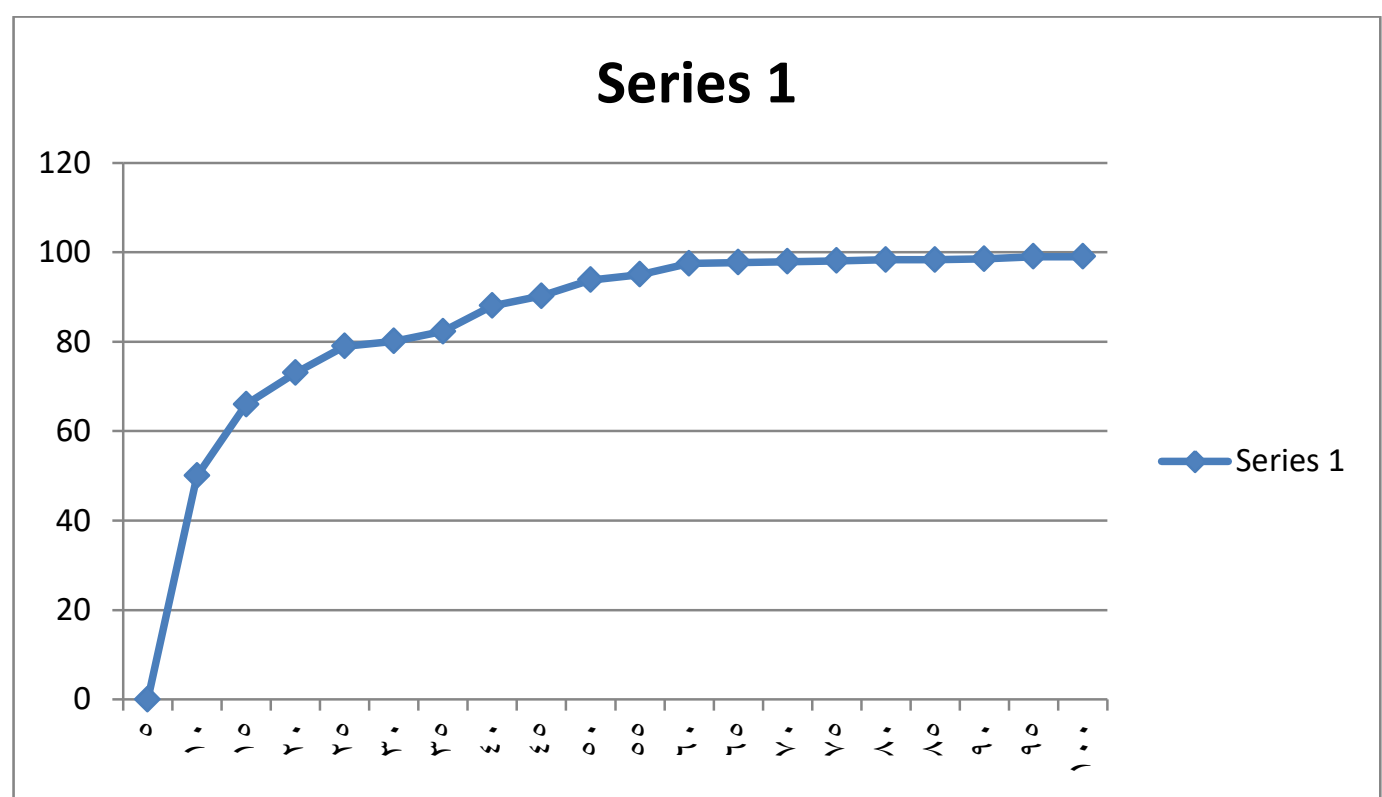

Fig. 3. Learning curve of chest ultrasound. 


\section{DISCUSSION}

In the critically ill patients, adequate treatment depends on early and accurate detection of problem, but the diagnosis is not always easy from the early moments of presentation. Shortly after initial resuscitation, ICU team should combine the results of physical examination, laboratory and radiological investigations rapidly to achieve accurate diagnosis, definitive treatment and best outcomes ${ }^{(6)}$. Although chest X-ray is the main imaging approach in many settings, many limitations exist for it e.g poor sensitivity. Chest CT scan remains the gold standard test in diagnosis of the most of chest diseases. Despite its higher diagnostic accuracy, it has many limitations. It is expensive, time consuming, impractical especially in the critically-ill patients, unsuitable with metallic prosthesis and has higher radiation exposure than chest X-rays ${ }^{(6,}$ 7)

From the traditional point of view, air has been considered as a major obstacle of ultrasound. For long time, the lung has been considered an organ non-amenable to examination with ultrasonography. The main principle of lung ultrasonography is reduction of lung aeration with different diseases resulting in changing the lung surface and generating distinct profiles or patterns characteristic for each (6). The main advantages of ultrasound are that it is a relatively inexpensive, broadly available, rapid procedure, easily learned, and free from ionizing radiation or contrast. Chest ultrasound has comparable results with chest CT in some diseases and can supplement other imaging modalities of the chest and guides a variety of diagnostic and therapeutic interventional procedures ${ }^{(8)}$.

Pleural effusion is a common problem in ICU patients with a variable range of incidence from $8 \%$ to $60 \%$. Correlation of results of physical examination and chest radiograph in spite of decreased sensitivity and specificity was the only available method for diagnosis. So a new modality in diagnosis with high sensitivity and specificity is needed especially with positioning limitations for chest $\mathrm{X}$-rays (9).
In our study, we found that, sensitivity, specificity and diagnostic accuracy of lung ultrasound in diagnosing pleural effusion were $95.4 \%, 97.1 \%$ and $96 \%$ respectively. While, sensitivity, specificity and diagnostic accuracy of chest X-rays in diagnosing pleural effusion were $70.7 \%, 91.45 \%$ and $78 \%$ respectively.

Our results were similar to the results obtained by El Mahalawy et al in 2017, including 130 mechanically ventilated and non-mechanically ventilated patients with thoracic ultrasound sensitivity of $94 \%$ and a specificity of $96 \%$ in comparison to $70 \%$ sensitivity and $90 \%$ specificity of chest $\mathrm{X}$ rays ${ }^{(6)}$.

In a meta-analysis done by Michiel Winkler et al in 2018, included 10 full text studies with 543 patients, with chest radiograph overall sensitivity of $49 \%$ (95\% CI, 40-58\%) and specificity of $92 \%$ (86-95\%). In seven studies of this meta-analysis, lung ultrasound overall sensitivity was $95 \%(92-96 \%)$ and specificity was $94 \%(90-97 \%)^{(10)}$.

The incidence of pneumothorax among mechanically ventilated patients is high and is considered as one of the most serious complications of positive pressure ventilation. Pneumothorax may be present in poly-trauma patients especially with chest trauma. However, unfortunately, it may be iatrogenic due to central venous catheter insertion, thoracocentesis and with positive pressure ventilation and all may necessitate chest tube insertion for drainage ${ }^{(6)}$.

Throughout our study we found that chest ultrasound had a sensitivity of $87.5 \%$, specificity of $98.5 \%$ and accuracy of $95 \%$ compared to $53.1 \%, 98.5 \%$ and $84 \%$ respectively for chest $\mathrm{X}$-rays in diagnosing pneumothorax.

The results in our study matched with El Gendy et al in 2018 on 192 patients. The chest ultrasound showed a considerable higher sensitivity than bedside chest X-rays ( $86.1 \%$ versus $52.7 \%$ ), it also showed higher, negative predictive values $(96.8 \%$ versus $90.1 \%)$, and diagnostic accuracy (95.3\% versus $90.6 \%$ ). However, chest X-rays had a slightly higher specificity than lung US (99.4\% versus $97.4 \%$ ), and higher positive predictive values $(95.0 \% \text { versus } 88.6 \%)^{(11)}$. 
In contrast to Hyacinthe et al in 2012 on 119 patients with chest trauma, sensitivity of chest ultrasound to pneumothorax was $53 \%{ }^{(12)}$.

Many meta-analysis studies showed results matching with our study results like Ding et al in 2011 with sensitivity and specificity of chest ultrasound of $88 \%$ and $99 \%$ respectively (13) and Alrajab et al in 2013 with US sensitivity and specificity of $78.6 \%$ and $98.4 \%$ respectively ${ }^{(14)}$.

Pneumonia has high incidence in ICU patients either newly developed or related to the cause of admission. Nowadays, lung ultrasound represents a dependable diagnostic modality for pneumonia ${ }^{(6)}$. In our study, Sensitivity and specificity of ultrasound was higher than chest X-rays in diagnosing pneumonia (89.3\% versus $60.7 \%)$ and $(97.7 \%$ versus $90.9 \%)$ respectively.

These results were similar to Nazerian et al in 2015 on 285 patients with sensitivity and specificity for diagnosing pneumonia significantly higher with ultrasound than chest X-ray (81\% versus $64 \%$ ) and (94\% versus 90\%) respectively. Also, our results coincided with Cortellaro et al in 2012 on 120 patients who stated that the sensitivity and specificity was significantly higher with ultrasound than chest X-ray (99\% versus $67 \%)$ and $(95 \%$ versus $85 \%)$ respectively ${ }^{(6,15,16)}$.

However on the other hand El khayat and Alam Eldeen in 2014 conducted a study on 62 patients and found that chest ultrasound was diagnostic in $46(74 \%)$ patients. This difference in accuracy might be attributed to the fact that transthoracic ultrasound technique allows identification of areas of consolidation only when they are connected to the pleural surface ${ }^{(17)}$.

Pulmonary edema is a life-threatening condition (either cardiogenic or noncardiogenic) that shows fluid accumulation in the lung parenchyma and air spaces impairing gas exchange which may be the cause of ICU admission or newly develop in the ICU. Although heart failure is common, there is considerable uncertainty about the incidence of acute cardiogenic pulmonary edema ${ }^{(18)}$.

Lung US offers a new tool for bedside diagnosis of pulmonary edema. In lung US, the B-line is always a comet-tail artifact, always arises from the pleural line and always moves in concert with lung-sliding. Briefly, air and water are simultaneously hit by ultrasound beams, as occurring when subpleural interlobular septa are edematous. Three or more B lines between two ribs are called lung-rockets ${ }^{(6)}$.

Throughout our study, sensitivity and specificity of chest ultrasound and chest Xrays in comparison to chest CT were $(88.9 \%$ versus $33.3 \%)$ and $(98.9 \%$ versus $98.9 \%)$ respectively.

Our results agreed with El Mahalawy et al in 2017 who stated that chest ultrasound sensitivity and specificity were $93 \%$ and $93 \%$ respectively. While, chest $\mathrm{X}$-rays sensitivity and specificity were $36 \%$ and $90 \%$ respectively ${ }^{(6)}$.

Another study conducted by Xirouchaki et al in 2011 on 42 mechanically ventilated patients scheduled for CT with prospectively studying them with a modified lung ultrasound protocol. They concluded that, sensitivity, specificity and accuracy of chest ultrasound in diagnosing interstitial syndrome were $94 \%, 93 \%$ and $94 \%$ respectively. While, sensitivity, specificity and accuracy of chest $\mathrm{X}$-rays in diagnosing interstitial syndrome were $46 \%, 80 \%$ and $58 \%$ respectively ${ }^{(19)}$.

In the current study, the overall sensitivity, specificity and diagnostic accuracy of chest ultrasound and chest X-rays of all population of the study, whatever diagnosis, were $(91.4 \%$ versus $61.7 \%)$, (98.3\% versus $96.2 \%)$ and (95.5\% versus $82.2 \%$ ) respectively.

These results matched with many studies e.g Agmy et al in 2018 who found the overall sensitivity and specificity of chest ultrasound were $93.2 \%$ and $100 \%$ respectively and Lichtenstein et al in 2015 who found the overall sensitivity and specificity of chest ultrasound ranging $90 \%$ and $100 \%$ respectively ${ }^{(20,21)}$.

Limitations of the study: This study has some limitations. Firstly, a relatively small number of patients were studied. However, we overcome this by diagnosing more than one disease in the same patient. Secondly, the time interval between lung ultrasound and CT could not be controlled, and was up to 4 hours in some cases. This might contribute to the observed discrepancy between methods. Thirdly, patients were positioned laterally for 
ultrasound examination, and this might change the localization of some abnormalities (i.e., pleural effusions). The use of the microconvex probe facilitates semi-posterior analyses with minimal or no patient mobilization. Finally, it is important to say that the lung ultrasound operator was not blind concerning clinical presentation of patients.

Conclusion: Chest ultrasound is reliable, quick, bedside, low-cost, non-invasive, nonionizing, more accurate, and easily educated for early detection of chest diseases and their follow up.

\section{Declaration of interest}

The authors report no conflicts of interest. The authors alone are responsible for the content and writing of the paper.

Funding information: None declared

\section{REFERENCES}

(1) Laura C. Robertson and Mohammed AlHaddad: Recognizing the critically ill patient. Anesth. Int. Care. Med. 2013; 14: 11-14.

(2) Lichtenstein DA and Mezière G: Relevance of Lung Ultrasound in the Diagnosis of Acute Respiratory Failure (The BLUE Protocol). CHEST. 2008; 134: 117-125.

(3) Lichtenstein DA: Classification of artifacts. Whole body ultra-sonography in the critically ill, Springer Heidelberg Dordrecht London New York, 1st edition, 2010, 185-188.

(4) Lichtenstein DA: BLUE protocol. Whole body ultrasonography in the critically ill, Springer Heidelberg Dordrecht London New York, 1st edition, 2010, 189-202.

(5) Austin JH, Muller NL, Friedman PJ, Hansell DM, Naidich DP, Remy-Jardin M, et al: Glossary of terms for CT of the lungs: recommendations of the nomenclature committee of the Fleischner society. Radiol. 1996; 10: 1148-1150.

(6) Ibrahim I. Elmahalawy, Nagwa M. Doha, Osama M. Ebeid, Mohammed A. AbdelHady, and Ola Saied: Role of thoracic ultrasound in diagnosis of pulmonary and pleural diseases in critically ill patients. Egy Chest Dis Tuber J, 2017; 66: 261266.
(7) Alzahran SA, Al-Salamah MA, AlMadani WH, and ElBarbary MA: Systematic review and meta-analysis for the use of ultrasound versus radiology in diagnosing of pneumonia. Crit Ultras $\mathrm{J}$. 2017; 9: 1-11.

(8) Remérand F, Dellamonica J, Mao Z, Ferrari F, Bouhemad B, Jianxin Y, et al: Multiplane ultrasound approach to quantify pleural effusion at the bedside, Inten Care Med. 2010; 36: 656-664.

(9) Maslove D, Chen BTM, Wang H, and Kuschner WG: The diagnosis and management of pleural effusions in the ICU. Inten Care Med. 2011; 28: 24-36.

(10) Winkler MH, Touw HR, Peter M. van de Ven, Twisk J, Tuinman PR: Diagnostic accuracy of chest radiograph, and when concomitantly studied lung ultrasound, in critically ill patients with respiratory symptoms: A systematic review and meta-analysis. Crit Care Med. 2018; 1-8.

(11)El Gendy M, Abdalla W, Abdelaziz AA, and Ammar MA: Lung ultrasound versus chest radiography for the diagnosis of pneumothorax in critically ill patients: A prospective, single-blind study. Saudi Anaes J. 2018; 265-269.

(12) Hyacinthe AC, Broux C, Francony G, Genty C, Bouzat P, Jacquot C, et al: Diagnostic accuracy of ultrasonography in the acute assessment of common thoracic lesions after trauma, Chest. 2012; 141: 1177-1179.

(13)Ding W, Shen Y, Yang J, He X, Zhang M: Diagnosis of pneumothorax by radiography and ultrasonography: a metaanalysis. Chest. 2011; 140(4): 859-866.

(14) Alrajab S, Youssef AM, Akkus NI, Caldito G: Pleural ultrasonography versus chest radiography for the diagnosis of pneumothorax: review of the literature and meta-analysis, Crit. Care. 2013; 17: 208- 212.

(15) Nazerian P, Volpicelli G, Vanni S, Gigli C, Betti L, Bartolucci M, et al: Accuracy of lung ultrasound for the diagnosis of consolidations when compared to chest computed tomography. Am J Emerg Med, 2015; 33: 620-625.

(16) Cortellaro F, Colombo S, Coen D, Duca PG: Lung ultrasound is an accurate 
diagnostic tool for the diagnosis of pneumonia in the emergency department. Emerg Med J. 2012; 29: 19-23.

(17) Al-khayat KF and Alam-Eldeen $\mathrm{MH}$ : Value of chest ultrasound in diagnosis of community acquired pneumonia. E J Chest Dis Tuber. 2014; 63(4): 10471051.

(18)Bourcier J-E, Paquet J, Seinger M, Gallard E, Redonnet J-P, Cheddadi F, et al: Performance comparison of lung ultrasound and chest $\mathrm{X}$-ray for the diagnosis of pneumonia in the ED. Am J Emerg Med. 2014; 32(2): 115-118.

(19)Xirouchaki N, Magkanas E, Vaporidi K, Kondili E, Plataki M, Patrianakos
A, Akoumianaki E, Georgopoulos D: Lung ultrasound in critically ill patients: comparison with bedside chest radiography. Inten Care Med. 2011; 37(9): 1488-1493.

(20) Agmy GR, Sherif H, Mohamed AS, Randa I, Aliaa AM: Assessment of severe dyspnea in critically ill patients by transthoracic sonography: Fayoum experience of the bedside lung ultrasonography in emergency protocol. Egy Bronchol J. 2018; 12: 92-97.

(21) Lichtenstein DA. BLUE-protocol and FALLS-protocol: two applications of lung ultrasound in the critically ill. Chest. 2015; 147: 779-789.

To Cite This Article: Nermeen MA, Hassan MA, Neven MG, Mohamed MT, Sameh SH. Evaluation Of The Role Of Bedside Lung Ultrasound Versus Chest X-Ray In Critically III Patients.ZUMJ 2019;25(6);887-897.DOi: 10.21608/zumj.2019.10704.11150. 